PROCEEDINGS OF THE

AMERICAN MATHEMATICAL SOCIETY

Volume 138, Number 4, April 2010, Pages 1213-1221

S 0002-9939(09)10178-8

Article electronically published on December 1, 2009

\title{
COVERS IN FINITELY ACCESSIBLE CATEGORIES
}

\author{
SEPTIMIU CRIVEI, MIKE PREST, AND BLAS TORRECILLAS
}

(Communicated by Birge Huisgen-Zimmermann)

\begin{abstract}
We show that in a finitely accessible additive category every class of objects closed under direct limits and pure epimorphic images is covering. In particular, the classes of flat objects in a locally finitely presented additive category and of absolutely pure objects in a locally coherent category are covering.
\end{abstract}

\section{INTRODUCTION}

In the theory of module approximation, originating in the work of Auslander and Smalø for finitely generated modules over finite dimensional algebras [3] and Enochs for modules over arbitrary rings [15], the Flat Cover Conjecture has played a special part. After Bican, El Bashir and Enochs gave a positive answer to the conjecture, showing that every module has a flat cover [5, Theorem 3], the problem of the existence of flat covers for any object was raised and solved in categories other than categories of modules over a ring. We point out the work of Aldrich, Enochs, García Rozas and Oyonarte [2] and El Bashir [14 in arbitrary Grothendieck categories; Enochs, Estrada, García Rozas and Oyonarte for quasi-coherent sheaves over the projective line 16 and for quasi-coherent sheaves over a scheme 17]; Enochs and Oyonarte [19] for modules over a sheaf of rings on a topological space; and Hovey [26] and Gillespie 23], 24] on flat model structures. The features of the categories considered have not usually allowed a straightforward generalization of the results established for modules, such as the key result of Eklof and Trlifaj [13, Theorem 10], which requires the existence of enough projectives. Therefore, specific techniques had to be developed in order to overcome such difficulties, and other features have been exploited, such as the existence of enough flat objects.

In the general theme of showing the existence of flat covers for any object in certain categories, we consider the situation for a locally finitely presented additive category. We shall prove a general result on covers in finitely accessible additive categories and then derive the existence of flat covers in locally finitely presented additive categories. The framework of an accessible category in the sense of [1] and,

Received by the editors April 17, 2009, and, in revised form, August 1, 2009.

2010 Mathematics Subject Classification. Primary 18E05, 18C35; Secondary 16D90.

Key words and phrases. Finitely accessible additive category, locally finitely presented additive category, (pre)cover, (pre)envelope, flat object, absolutely pure object.

This work was partially supported by MEC Romania (grant PN-II-ID-PCE-2008-2 project ID_2271), MEC Spain and DGI Spain (project MTM2008-03339), and Junta de Andalucía (Proyecto de Excelencia FQM 3128). The first author would like to thank the Department of Algebra and Analysis for their kind hospitality during his stays at the University of Almería.

(C)2009 American Mathematical Society Reverts to public domain 28 years from publication 
in particular, of a finitely accessible additive category [6], is a natural one in which to consider purity. Any finitely accessible additive category $\mathcal{C}$ may be embedded as a full subcategory of the category $\operatorname{Mod}(A)$ of unitary right $A$-modules, where $A$ is a ring with enough idempotents, termed the functor ring of $\mathcal{C}$, such that the pure exact sequences in $\mathcal{C}$ are those which become exact sequences in $\operatorname{Mod}(A)$ through the embedding (e.g., see [6, [12]). Then $\mathcal{C}$ may be seen as being equivalent to the full subcategory $\operatorname{Fl}(A)$ of the category $\operatorname{Mod}(A)$ consisting of flat right $A$-modules. The category $\operatorname{Mod}(A)$ also is equivalent to the functor category $\left(\mathrm{fp}(\mathcal{C})^{\mathrm{op}}, \mathrm{Ab}\right)$ of all contravariant additive functors from the full subcategory $\operatorname{fp}(\mathcal{C})$ of finitely presented objects of $\mathcal{C}$ to the category $\mathrm{Ab}$ of abelian groups, and this equivalence restricts to one between the full subcategories of flat objects of $\operatorname{Mod}(A)$ and $\left(f \mathrm{fp}(\mathcal{C})^{\mathrm{op}}, \mathrm{Ab}\right)$. Functor ring (functor category) techniques may be employed in order to relate properties of modules over rings with enough idempotents (objects in a functor category) to properties of objects in finitely accessible additive categories (e.g., see [12], 20]). Concerning module approximation theory, we recall that Herzog [25] used such an approach to prove the existence of a pure-injective envelope for any object in a finitely accessible additive category.

A similar basic idea and a general result on the existence of covers for modules over rings with enough idempotents will be the key ingredients in showing our main result, namely: If $\mathcal{C}$ is a finitely accessible additive category and $\mathcal{A}$ is a class of objects of $\mathcal{C}$ closed under direct limits and pure epimorphic images, then every object of $\mathcal{C}$ has an $\mathcal{A}$-cover. Also, if $\mathcal{D}$ is a definable category and $\mathcal{A}$ is a class of objects of $\mathcal{D}$ closed under direct limits and pure epimorphic images, we show that every object of $\mathcal{D}$ has an $\mathcal{A}$-cover. As a consequence, we solve Open Problem 2.9 (b) from [10, which asks for a characterization of finitely accessible Grothendieck categories such that every object has a flat cover. Our result applies, for instance, to the class of flat objects in the category of comodules over an arbitrary coalgebra. Moreover, we show that every object of a locally coherent category has an absolutely pure cover.

Now let us recall some necessary terminology. Throughout, $\mathcal{C}$ will be an additive category. The category $\mathcal{C}$ is said to be finitely accessible ("locally finitely presented" in the terminology of [6]) if it has direct limits, the class of finitely presented objects is skeletally small, and every object is a direct limit of finitely presented objects 11. Suppose that $\mathcal{C}$ is finitely accessible. A sequence $0 \rightarrow X \stackrel{f}{\rightarrow} Y \stackrel{g}{\rightarrow} Z \rightarrow 0$ of morphisms in $\mathcal{C}$ is pure exact if it induces an exact sequence of abelian groups $0 \rightarrow \operatorname{Hom}_{\mathcal{C}}(P, X) \rightarrow \operatorname{Hom}_{\mathcal{C}}(P, Y) \rightarrow \operatorname{Hom}_{\mathcal{C}}(P, Z) \rightarrow 0$ for every finitely presented object $P$ of $\mathcal{C}$ [6]. This implies that $g, f$ form a kernel-cokernel pair, that $f$ is a monomorphism and that $g$ is an epimorphism. In such a pure-exact sequence $f$ is said to be a pure monomorphism and $g$ a pure epimorphism.

By a class of objects in an additive category $\mathcal{C}$ we always mean a class of objects closed under isomorphisms. Let $M$ be an object in $\mathcal{C}$ and $\mathcal{X}$ a class of objects in $\mathcal{C}$. Recall (e.g., see [37]) that a morphism $f \in \operatorname{Hom}(X, M)$, with $X \in \mathcal{X}$, is an $\mathcal{X}$ precover of $M$ if the induced abelian group morphism $\operatorname{Hom}\left(X^{\prime}, X\right) \rightarrow \operatorname{Hom}\left(X^{\prime}, M\right)$ is surjective for every $X^{\prime} \in \mathcal{X}$. An $\mathcal{X}$-precover $f \in \operatorname{Hom}(X, M)$ of $M$ is an $\mathcal{X}$-cover if every endomorphism $g: X \rightarrow X$ with $f g=f$ is an automorphism. The class $\mathcal{X}$ is called (pre)covering if every object of $\mathcal{C}$ has an $\mathcal{X}$-cover. In a dual manner one defines the notions of relative (pre)envelope and (pre)enveloping class. 
If $\mathcal{C}$ is a finitely accessible category with products, then a full subcategory $\mathcal{D}$ which is closed under products, direct limits and pure subobjects is said to be a definable subcategory. These classes arose in the model theory of modules (see for example [31]), and the terminology was introduced by Crawley-Boevey [6]. The categories which arise in this way are precisely the exactly definable categories in the sense of Krause [28]. For a general treatment of these categories see [32].

\section{Covers}

Over a ring with identity, every class of modules which is closed under direct sums and pure epimorphic images is covering. This result, Theorem 2.4 below, can be obtained from the arguments in [5] and is explicitly stated in [4, Theorem 2.5] and [27, Theorem 2.5]. By, for instance [36, 33.9], a class of modules is closed under direct sums and pure epimorphic images if and only if it is closed under direct limits and pure epimorphic images. We first extend the above result to modules over a ring with enough idempotents, giving a proof based on an idea, different from that of [27, employed in 30] for showing that the class of absolutely pure modules over a right coherent ring is covering.

Throughout this section $R=\bigoplus_{i \in I} e_{i} R=\bigoplus_{i \in I} R e_{i}$ is a ring with enough idempotents and all modules are unitary right $R$-modules. First we need to recall two results. The first is proved in 5 for modules over a ring with identity, but the proof is almost the same as in the case of a ring with enough idempotents. Alternatively one may extend to a ring with identity and deduce the more general result from that case. Recall that a purity $\sigma$ is projectively generated by a class $\mathcal{A}$ of modules if the $\sigma$-pure sequences are exactly those with respect to which every $A \in \mathcal{A}$ is projective.

Theorem 2.1 ([5, Theorem 5]). Let $\sigma$ be a purity projectively generated by a set of modules. Then for each cardinal $\lambda$ there is a cardinal $\kappa$ such that for any module $N$ with $|N| \geq \kappa$ and for any submodule $L$ of $N$ with $|N / L| \leq \lambda, L$ contains a non-zero $\sigma$-pure submodule of $N$.

Lemma 2.2 ([30, Lemma 2.4]). Let $M$ be a module, $\mathcal{A}$ a class of modules closed under direct sums and $\mathcal{B} \subset \mathcal{A}$ a set such that any homomorphism $A \rightarrow M$ with $A \in \mathcal{A}$ factors through a module in $\mathcal{B}$. Then $M$ has an $\mathcal{A}$-precover.

Proof. One checks that the canonical map $\bigoplus_{B \in \mathcal{B}} B^{(\operatorname{Hom}(B, M))} \rightarrow M$ is an $\mathcal{A}$ precover of $M$.

Suppose that $\mathcal{C}$ is the category of modules over a ring with enough idempotents; then there is, up to isomorphism, just a set of finitely presented modules. The usual notion of purity is that which is projectively generated by the set (to isomorphism) of finitely presented modules, so Theorem 2.1] applies in this situation.

Lemma 2.3. Let $\mathcal{A}$ be a class of modules (over a ring with enough idempotents) closed under direct limits and pure epimorphic images. Let $M$ be a module with $|M|=\lambda$ and let $\kappa$ be the cardinal from Theorem 2.1. Then any homomorphism $A \rightarrow M$ with $A \in \mathcal{A}$ factors through a module $B \in \mathcal{A}$ with $|B|<\kappa$.

Proof. Let $f_{0}: A \rightarrow M$ be a homomorphism with $A \in \mathcal{A}$ having cardinality $\geq \kappa$ and let $K_{0}=\operatorname{Ker}\left(f_{0}\right)$. Since $\left|A / K_{0}\right| \leq|M|,\left|A / K_{0}\right| \leq \lambda$; hence $K_{0}$ contains a non-zero pure submodule $L_{0}$ of $A$ by Theorem 2.1. Then $A / L_{0} \in \mathcal{A}$. If $A / L_{0}$ does 
not have cardinality $<\kappa$, then repeat the process with the induced homomorphism $f_{1}: A / L_{0} \rightarrow M$ in place of $f_{0}$. Let $K_{1} / L_{0}=\operatorname{Ker}\left(f_{1}\right)$. Since $\left|A / K_{1}\right| \leq \lambda, K_{1} / L_{0}$ contains a non-zero pure submodule $L_{1} / L_{0}$ of $A / L_{0}$ by Theorem 2.1] Then $A / L_{1} \in$ $\mathcal{A}$. If $\left|A / L_{1}\right|$ is not $<\kappa$, then continue the process with the induced homomorphism $f_{2}: A / L_{1} \rightarrow M$. Continue transfinitely, setting $L_{\gamma}=\bigcup_{\alpha<\gamma}$ if $\gamma$ is a limit ordinal and noting that $A / L_{\gamma}=\lim _{\alpha<\gamma} A / L_{\alpha}$ is in $\mathcal{A}$ by assumption. Eventually we reach some $\alpha$ with $B=A / L_{\alpha} \in \mathcal{A}$, and $|B|<\kappa$; hence the required factorization.

Theorem 2.4. Let $\mathcal{A}$ be a class of modules (over a ring with enough idempotents) closed under direct limits and pure epimorphic images. Then $\mathcal{A}$ is covering.

Proof. Since $\mathcal{A}$ is closed under direct limits, in order to derive the conclusion it is enough to show that every module has an $\mathcal{A}$-precover [37, Theorem 2.2.8]. Let $M$ be a module and set $\lambda=|M|$. Let $\kappa$ be the cardinal from Theorem 2.1. Note that there is, up to isomorphism, just a set $\mathcal{B}$ of modules of cardinality $<\kappa$.

We claim that $\bigoplus_{B \in \mathcal{B}} B^{(\operatorname{Hom}(B, M))} \rightarrow M$ is an $\mathcal{A}$-precover of $M$. Let $f: A \rightarrow M$ be a homomorphism with $A \in \mathcal{A}$. By Lemma 2.3, $f$ factors through a module $B \in \mathcal{A}$ with $|B|<\kappa$, and so through a module in $\mathcal{A}$ isomorphic to one in $\mathcal{B}$. Now by Lemma 2.2 it follows that $\bigoplus_{B \in \mathcal{B}} B^{(\operatorname{Hom}(B, M))} \rightarrow M$ is an $\mathcal{A}$-precover of $M$.

In what follows, we shall further extend Theorem 2.4 to the framework of a finitely accessible additive category. We shall need a property on functors reflecting covers. For its proof, we recall from [29] the concept of a separable functor. Let $F: \mathcal{C} \rightarrow \mathcal{D}$ be a covariant functor between (additive) categories and consider the associated natural transformation $\mathcal{F}: \operatorname{Hom}_{\mathcal{C}}(-,-) \rightarrow \operatorname{Hom}_{\mathcal{D}}(F(-), F(-))$ defined by $\mathcal{F}_{C, C^{\prime}}(f)=F(f)$ for every morphism $f: C \rightarrow C^{\prime}$ in $\mathcal{C}$. The functor $F$ is called separable if $\mathcal{F}$ splits as a natural transformation; that is, there is a natural transformation $\mathcal{L}: \operatorname{Hom}_{\mathcal{D}}(F(-), F(-)) \rightarrow \operatorname{Hom}_{\mathcal{C}}(-,-)$ such that $\mathcal{L} \circ \mathcal{F}=1_{\operatorname{Hom}_{\mathcal{C}}(-,-)}$.

Lemma 2.5. Let $F: \mathcal{C} \rightarrow \mathcal{D}$ be a covariant full and faithful functor between additive categories with $\mathcal{A}$ and $F(\mathcal{A})$ full subcategories of $\mathcal{C}$ and $\mathcal{D}$ respectively. If $F(\mathcal{A})$ is covering in $\mathcal{D}$, then $\mathcal{A}$ is covering in $\mathcal{C}$.

Proof. A direct proof is straightforward. Alternatively, note that every full and faithful functor is separable; hence the result basically follows by [22, Proposition 2], which holds in arbitrary additive categories.

Theorem 2.6. Let $\mathcal{C}$ be a finitely accessible category and $\mathcal{A}$ a class of objects of $\mathcal{C}$ closed under direct limits and pure epimorphic images. Then $\mathcal{A}$ is covering.

Proof. The equivalence between $\mathcal{C}$ and the full subcategory of $\left(f p(\mathcal{C})^{\mathrm{op}}, \mathrm{Ab}\right)$ consisting of the flat objects is induced by the covariant Yoneda functor $H: \mathcal{C} \rightarrow$ $\left(\mathrm{fp}(\mathcal{C})^{\mathrm{op}}, \mathrm{Ab}\right)$, which sends an object $Z$ of $\mathcal{C}$ to the functor $H_{Z}=\left.\operatorname{Hom}(-, Z)\right|_{\mathrm{fp}(\mathcal{C})}$, and a morphism $f: X \rightarrow Y$ in $\mathcal{C}$ to the natural transformation $H_{f}=\operatorname{Hom}(-, f)$ : $H_{X} \rightarrow H_{Y}$ [6, 1.4 Theorem]. Recall that we have an equivalence between the categories $\left.(f p(C))^{\mathrm{op}}, \mathrm{Ab}\right)$ and $\operatorname{Mod}(A)$ of unitary right modules over the functor $\operatorname{ring} A$ of $\mathcal{C}$. Since $H$ is full and faithful, in light of Theorem 2.4 and Lemma 2.5, it suffices to show that $H(\mathcal{A})$ has the same closure properties in $\left(\mathrm{fp}(\mathcal{C})^{\mathrm{op}}, \mathrm{Ab}\right)$ as those assumed of $\mathcal{A}$ in $\mathcal{C}$. Note that $H(\mathcal{A})$ is closed under direct limits, because $H$ commutes with direct limits. Let $0 \rightarrow K \rightarrow H_{Y} \rightarrow N \rightarrow 0$ be a pure exact sequence in $\left(\operatorname{fp}(\mathcal{C})^{\mathrm{op}}, \mathrm{Ab}\right)$ with $Y \in \mathcal{A}$. Then both $K$ and $N$ have to be flat (e.g., see [32, Proposition 5.9] and [36, 36.1]), and so $K \cong H_{X}$ and $N \cong H_{Z}$ for some objects $X$ and $Z$ of $\mathcal{C}$. Since 
$H$ is full we have the induced pure exact sequence $0 \rightarrow X \rightarrow Y \rightarrow Z \rightarrow 0$ in $\mathcal{C}$ [6]. By hypothesis, we have $Z \in \mathcal{A}$; hence $N \in H(\mathcal{A})$. Therefore, $H(\mathcal{A})$ is closed under pure epimorphic images.

Now let $\mathcal{C}$ be a finitely accessible category with products and let $\mathcal{D}$ be a definable subcategory of $\mathcal{C}$. Note that definable subcategories need not be finitely accessible: for instance, the category of divisible abelian groups is a definable subcategory of the category Ab of abelian groups, but it is not finitely accessible [32, Example 10.3]. Nevertheless, we may establish our covering result in such a context because of the good behaviour with respect to purity, namely: purity in a definable subcategory is just the restriction of purity in the larger category (see, e.g., 32] for details).

Theorem 2.7. Let $\mathcal{D}$ be a definable subcategory of a finitely accessible category with products and let $\mathcal{A}$ be a class of objects of $\mathcal{D}$ closed under direct limits and pure epimorphic images. Then $\mathcal{A}$ is covering.

Proof. Note that $\mathcal{D}$ may be seen as a definable subcategory of a functor category [32, Proposition 10.1]. Now a proof such as that for Theorem 2.6 works.

Recall that the definable categories - those which are, up to equivalence, definable subcategories of finitely accessible additive categories with products - are (32, Proposition 11.1]) precisely the exactly definable categories in the sense of [28, meaning those equivalent to the category $\operatorname{Ex}\left(\mathcal{B}^{\circ p}, \mathrm{Ab}\right)$ of exact additive functors from a skeletally small abelian category $\mathcal{B}^{\text {op }}$ to $\mathrm{Ab}$.

\section{Applications}

Throughout this section $\mathcal{C}$ will be a finitely accessible additive category. We begin by considering notions of flatness and absolute purity in $\mathcal{C}$ which generalize flatness and absolute purity in the sense of Stenström [35, given in finitely accessible Grothendieck categories.

Definition 3.1. An object $Z$ of $\mathcal{C}$ is said to be flat if every epimorphism $Y \rightarrow Z$ in $\mathcal{C}$ is pure. An object $X$ of $\mathcal{C}$ is said to be absolutely pure if every monomorphism $X \rightarrow Y$ in $\mathcal{C}$ is pure.

Example 3.2. Let us observe that our notions of flatness and purity are different from those considered in [19] for modules over a sheaf of rings on a topological space. Indeed those notions as usually defined for sheaves of modules are not the categorically defined ones.

Let $K$ be a field and consider the path algebra $K A_{2}$ where $A_{2}$ is the quiver $1 \stackrel{\alpha}{\rightarrow} 2$ (thus $K A_{2}$ may be regarded as the ring of upper-triangular $2 \times 2$ matrices over $K$ ). The category of right $K A_{2}$-modules is equivalent to the category of $K$-representations of $A_{2}$; it is also equivalent to the category Mod- $\mathcal{O}$ where $\mathcal{O}$ is the ringed space defined as follows. Take the topological space to be $T=\{1,2\}$ equipped with the topology which has $T,\{2\}, \emptyset$ as open sets. Define $\mathcal{O}$ by $\mathcal{O}(T)=$ $K, \mathcal{O}(\{2\})=K$ and with the restriction map being the identity map $1_{K}$. It is easy to see (e.g., [33, Ex. 2.1]) that every presheaf of $\mathcal{O}$-modules is a sheaf and that the category Mod- $O$ is equivalent to Mod- $\mathrm{KA}_{2}$; in particular it is locally finitely presented.

If we take the usual, pointwise, definitions of purity and flatness for sheaves of modules, then it is immediate that every sheaf is flat and every embedding is pure 
under those definitions simply because that is the case in the category Mod- $K$. Yet the embedding of the representation $0 \stackrel{0}{\rightarrow} K$ into $K \stackrel{1}{\rightarrow} K$ is not pure in the category Mod- $K A_{2}$, nor is the representation $K \stackrel{0}{\rightarrow} 0$ a flat object of that category.

The point is that if $\mathcal{O}$ is a sheaf of rings, then there are two natural tensor product structures on the category Mod- $\mathcal{O}$ of $\mathcal{O}$-modules. The "sheaf" definition is to use the pointwise monoidal structures (that is, the pointwise tensor product) on the categories of modules over the stalks of $\mathcal{O}$. On the other hand, to every definable category $\mathcal{D}$ is associated a "dual" definable category, $\mathcal{D}^{\mathrm{d}}$, such that one may define a tensor product $\mathcal{D} \times \mathcal{D}^{\mathrm{d}} \rightarrow \mathrm{Ab}$ (see [32, §9]). If $\mathcal{D}=\operatorname{Mod}-R$ for some ring $R$ (with enough idempotents), then the dual category $\mathcal{D}^{\mathrm{d}}$ is just $R$-Mod and we have the usual tensor product. If, however, $\mathcal{D}$ is a category of sheaves, even of commutative rings, then the dual construction "reverses" the topology, as can be seen in the example above, and so the categorical tensor product, defined using the dual category, is in general different from that defined for sheaves.

The next result extends [10, Theorem 2.6] to locally finitely presented additive categories. Recall that an additive category is locally finitely presented if it is finitely accessible and cocomplete (i.e., has all colimits) or, equivalently, if it is finitely accessible and complete (i.e., has all limits) 32 .

Corollary 3.3. Let $\mathcal{C}$ be a locally finitely presented category. Then the class of flat objects of $\mathcal{C}$ is covering. In particular, the class of flat objects in a finitely accessible Grothendieck category is covering.

Proof. The existence of pullbacks (and kernels) in $\mathcal{C}$ allows one to show, as for modules (e.g., see [36, 36.1]), that the class of flat objects of $\mathcal{C}$ is closed under direct limits and pure epimorphic images. Then use Theorem 2.6 .

Let us recall that there are finitely accessible Grothendieck categories not having enough flat objects and even without any non-zero flat object. For instance, if $Q$ is the infinite quiver $\bullet \rightarrow \bullet \rightarrow \ldots, K$ is a field, and $\operatorname{Rep}_{K}(Q)$ denotes the Grothendieck $K$-category of all $K$-linear representations of $Q$, then the full $K$ subcategory of $\operatorname{Rep}_{K}(Q)$ consisting of all locally finite dimensional representations (that is, directed unions of finite dimensional representations) has no non-zero flat object [10, Example 2.10].

Now we are able to extend the existence of flat covers for comodules over a semiperfect coalgebra [10, Theorem 3.9] (also see [8, Corollary 2.5]) to comodules over an arbitrary coalgebra provided the category of comodules is finitely accessible Grothendieck. In particular, this applies to the locally finitely presented Grothendieck category of right $C$-comodules when $C$ is a coalgebra over a field $k$ [1].

Corollary 3.4. Let $K$ be a field and $C$ be a coalgebra over $K$. Then the class of flat right $C$-comodules is covering.

The following result generalizes [30, Theorem 2.6].

Corollary 3.5. Let $\mathcal{C}$ be a locally coherent category. Then the classes of absolutely pure objects of $\mathcal{C}$ and of absolutely pure flat objects of $\left(\mathrm{fp}(\mathcal{C})^{\mathrm{op}}, \mathrm{Ab}\right)$ are covering.

Proof. Since $\mathcal{C}$ is locally coherent, the functor ring $A$ of $\mathcal{C}$ is two-sided panoramic [20. Proposition 2.4] in the sense that the additive categories of flat unitary right 
modules and flat unitary left modules over the functor ring $A$ of $\mathcal{C}$ are abelian [21. In particular, $A$ is right and left locally coherent [21, Corollary 2.11]. Then (see [32, Proposition 5.8]) the class of absolutely pure objects of $\mathcal{C}$ is definable, so closed under direct limits and is, moreover, closed under pure epimorphic images. Also, note that absolutely pure objects in $\mathcal{C}$ are in one-to-one correspondence with absolutely pure flat objects of $\left(\mathrm{fp}(\mathcal{C})^{\mathrm{op}}, \mathrm{Ab}\right)$ by the proof of 9 , Proposition 2.1]. Now use Theorem 2.6.

\section{Preenvelopes}

In a similar way one shows the following result of a dual flavour, which generalizes a module-theoretic property considered by Rada and Saorín [34, Corollary 3.5].

Theorem 4.1. If $\mathcal{C}$ is a finitely accessible additive category with products and $\mathcal{A}$ is a class of objects of $\mathcal{C}$ closed under products and pure subobjects, then $\mathcal{A}$ is a preenveloping class.

Proof. We only sketch the proof. First, one shows that over a ring with enough idempotents $R$, any class of unitary right $R$-modules closed under products and pure submodules is preenveloping. This follows in the same way as the similar result over a ring with identity [34, Corollary 3.5], which in turn is based on the basically categorical proof of [34, Theorem 3.3]. Second, note that if $F: \mathcal{C} \rightarrow \mathcal{D}$ is a covariant full and faithful functor between additive categories with $\mathcal{A}$ and $F(\mathcal{A})$ full subcategories of $\mathcal{C}$ and $\mathcal{D}$ respectively and $F(\mathcal{A})$ is preenveloping in $\mathcal{D}$, then $\mathcal{A}$ is preenveloping in $\mathcal{C}$. Finally, as in Theorem [2.6, one considers the covariant Yoneda functor $H: \mathcal{C} \rightarrow\left(\operatorname{fp}(\mathcal{C})^{\mathrm{op}}, \mathrm{Ab}\right)$. Note that if $\mathcal{C}$ has products, then $\mathcal{C}$, which is equivalent to the full subcategory of flat objects of $\left(\operatorname{fp}(\mathcal{C})^{\mathrm{op}}, \mathrm{Ab}\right)$, may be viewed as being closed under products in $\left(f p(\mathcal{C})^{\mathrm{op}}, \mathrm{Ab}\right)$ 6, Theorem 2.1]. Then it follows that $H(\mathcal{A})$ is closed under products. Arguments such as those used in the proof of Theorem 2.6 for proving closure under pure epimorphic images allow one to show that $H(\mathcal{A})$ is closed under pure subobjects. Now the result follows.

In particular, the classes of absolutely pure objects in a locally finitely presented additive category and of flat objects in a locally coherent category are closed under products and pure subobjects (see Propositions 5.8 and 5.9, and Theorem 6.1 from [32]). Then we obtain the following generalization of [18, Proposition 6.2.4] and [18, Proposition 6.5.1].

Corollary 4.2. The classes of absolutely pure objects in a locally finitely presented additive category and of flat objects in a locally coherent category are preenveloping.

\section{REFERENCES}

1. J. Adámek and J. Rosický, Locally presentable and accessible categories, London Math. Soc., Lecture Note Ser., 189, Cambridge University Press, 1994. MR.1294136 (95j:18001)

2. S.T. Aldrich, E.E. Enochs, J.R. García Rozas and L. Oyonarte, Covers and envelopes in Grothendieck categories. Flat covers of complexes with applications, J. Algebra 243 (2001), 615-630. MR1850650 (2002i:18010)

3. M. Auslander and S.O. Smalø, Preprojective modules over Artin algebras, J. Algebra 66 (1980), 61-122. MR591246 (83a:16039)

4. S. Bazzoni, When are definable classes tilting and cotilting classes?, J. Algebra 320 (2008), 4281-4299. MR2464106 (2009i:16001)

5. L. Bican, R. El Bashir and E. Enochs, All modules have flat covers, Bull. London Math. Soc. 33 (2001), 385-390. MR1832549 (2002e:16002) 
6. W. Crawley-Boevey, Locally finitely presented additive categories, Comm. Algebra 22 (1994), 1641-1674. MR1264733 (95h:18009)

7. W. Crawley-Boevey, Infinite-dimensional modules in the representation theory of finitedimensional algebras, pp. 29-54 in I. Reiten, S. Smaløand, Ø. Solberg (eds.), Algebras and Modules. I, Canadian Math. Soc. Conf. Proc., Vol. 23, Amer. Math. Soc., Providence, RI, 1998. MR 1648602 (99m:16016)

8. S. Crivei, A note on flat covers of comodules, Mathematica (Cluj) 50 (73) (2008), 187-190.

9. S. Crivei and J.L. García, Gruson-Jensen duality for idempotent rings, Comm. Algebra 33 (2005), 3949-3966. MR2183972 (2007f:16009)

10. J. Cuadra and D. Simson, Flat comodules and perfect coalgebras, Comm. Algebra 35 (2007), 3164-3194. MR2356149 (2008j:16108)

11. S. Dăscălescu, C. Năstăsescu and Ş. Raianu, Hopf Algebras. An Introduction, Marcel Dekker, New York, 2001. MR1786197(2001j:16056)

12. N.V. Dung and J.L. García, Additive categories of locally finite representation type, J. Algebra 238 (2001), 200-238. MR1822190 (2002f:18021)

13. P.C. Eklof and J. Trlifaj, How to make Ext vanish, Bull. London Math. Soc. 33 (2001), 41-51. MR.1798574 (2001i:16015)

14. R. El Bashir, Covers and directed colimits, Algebr. Represent. Theory 7 (2004), 423-430. MR 2252654 (2007k:16003)

15. E.E. Enochs, Injective and flat covers, envelopes and resolvents, Israel J. Math. 39 (1981), 189-209. MR636889 (83a:16031)

16. E.E. Enochs, S. Estrada, J.R. García Rozas and L. Oyonarte, Flat covers in the category of quasi-coherent sheaves over the projective line, Comm. Algebra 32 (2004), 1497-1508. MR 2100370 (2005m:14024)

17. E.E. Enochs, S. Estrada, J.R. García Rozas and L. Oyonarte, Flat and cotorsion quasicoherent sheaves. Applications, Algebr. Represent. Theory 7 (2004), 441-456. MR2096670 (2005i:14019)

18. E.E. Enochs and O.M.G. Jenda, Relative homological algebra, de Gruyter, Berlin, 2000. MR.1753146 (2001h:16013)

19. E.E. Enochs and L. Oyonarte, Flat covers and cotorsion envelopes of sheaves, Proc. Amer. Math. Soc. 130 (2001), 1285-1292. MR1879949 (2003d:18023)

20. J.L. García, P.L. Gómez Sánchez and J. Martínez Hernández, Locally finitely presented categories and functor rings, Osaka J. Math. 42 (2005), 173-187. MR.2132009(2006a:16013)

21. J.L. García and D. Simson, On rings whose flat modules form a Grothendieck category, Colloq. Math. 73 (1997), 115-141. MR1436954 (98a:16002)

22. J.R. García Rozas and B. Torrecillas, Preserving and reflecting covers by functors. Applications to graded modules, J. Pure Appl. Algebra 112 (1996), 91-107. MR.1402398 (97k:18011)

23. J. Gillespie, The flat model structure on $\mathrm{Ch}(R)$, Trans. Amer. Math. Soc. 356 (2004), 33693390. MR2052954 (2005b:18020)

24. J. Gillespie, The flat model structure on complexes of sheaves, Trans. Amer. Math. Soc. 358 (2006), 2855-2874. MR2216249 (2007a:55024)

25. I. Herzog, Pure-injective envelopes, J. Algebra Appl. 4 (2003), 397-402. MR2020947 (2004i:18003)

26. M. Hovey, Cotorsion pairs, model category structures, and representation theory, Math. Z. 241 (2002), 553-592. MR1938704 (2003m:55027)

27. P. Jørgensen and H. Holm, Covers, precovers, and purity, Illinois J. Math. 52 (2008), 691-703.

28. H. Krause, Exactly definable categories, J. Algebra 201 (1998), 456-492. MR1612398 (99j:18010)

29. C. Năstăsescu, M. Van den Bergh and F. Van Oystaeyen, Separable functors applied to graded rings, J. Algebra 123 (1989), 397-413. MR.1000494(90j:16001)

30. K.R. Pinzon, Absolutely pure covers, Comm. Algebra 36 (2008), 2186-2194. MR2418384 (2009i:16004)

31. M. Prest, Purity, spectra and localisation, Cambridge University Press, 2009.

32. M. Prest, Definable additive categories: Purity and model theory, Mem. Amer. Math. Soc., to appear. MIMS EPrint: 2006.218, July 2006.

33. M. Prest, V. Puninskaya and A. Ralph, Some model theory of sheaves of modules, J. Symbolic Logic 69 (2004), 1187-1199. MR2135662 (2005m:18003) 
34. J. Rada and M. Saorín, Rings characterized by (pre)envelopes and (pre)covers of their modules, Comm. Algebra 26 (1998), 899-912. MR.1606190 (99f:16002)

35. B. Stenström, Purity in functor categories, J. Algebra 8 (1968), 352-361. MR0229697 $(37: 5271)$

36. R. Wisbauer, Foundations of module and ring theory, Gordon and Breach, Reading, MA, 1991. MR 1144522 (92i:16001)

37. J. Xu, Flat covers of modules, Lecture Notes in Math., 1634, Springer, Berlin, 1996. MR:1438789 (98b:16003)

Faculty of Mathematics and Computer Science, "Babeş-Bolyai" University, Str. Mihail Kogălniceanu 1, 400084 Cluj-Napoca, Romania

E-mail address: crivei@math.ubbcluj.ro

School of Mathematics, Jan Turing Bullding, University of Manchester, Oxford Road, Manchester M13 9PL, United Kingdom

E-mail address: mprest@manchester.ac.uk

Departamento de Álgebra y Análisis, Universidad de Almería, 04071 Almería, Spain

E-mail address: btorreci@ual.es 